\title{
Hume en Deleuze: los primeros lineamientos del empirismo trascendental
}

\author{
Hume in Deleuze: the first outlines of \\ transcendental empiricism
}

\author{
Julien CANAVERA ${ }^{1}$
}

(Universitat de València)

Recibido: $28 / 10 / 2011$

Aceptado: 19/04/2012

\section{Resumen}

Deleuze gustaba de parangonar su quehacer filosófico con una suerte de patchwork o collage: un pensamiento al estilo Arlequín, abigarrado y hecho de fragmentos no totalizables. Tal es el pluralismo (o empirismo) reivindicado por el filósofo francés, y también su resultado: un bizarro mosaico hecho de encuentros o téléscopages entre autores aparentemente no relacionados. No obstante, el caso de Empirismo y subjetividad (1953) resulta un tanto extraño: no parece encajar en este complejo puzzle, y la infravaloración de la que adolece en numerosos comentarios sobre Deleuze tiende a reforzar ese prejuicio. Por el contrario, nos proponemos mostrar que este estudio sobre Hume contiene las primeras secuencias de una metafísica que quedará plenamente establecida en Diferencia y repetición -"l'oeuvre souche"- y, en paralelo, cómo esta temática naciente del Afuera hará surgir la temática complementaria de la Implicación -en otras palabras, la superación del fisicalismo humeano.

Palabras clave: Hume, Deleuze, Empirismo, subjetivación, hábito.
Abstract
Deleuze liked to compare his philosophical work with a kind of patchwork or collage: Harlequin-style thinking, colourful and made of not holistic fragments. This is the pluralism (or empiricism) claimed by the French philosopher, and its
1 Doctorando en Filosofía por la Universitat de València. Becario UVEG “Cinc Segles”. 
result: an unusual mosaic made of encounters or téléscopages between seemingly unrelated authors. However, the case of Empiricism and Subjectivity (1953) is rather strange: it does not seem to fit in this complex puzzle, and the undervaluation suffered in numerous comments on Deleuze tends to reinforce this prejudice. On the contrary, we intend to show that this study about Hume contains the first sequences of a metaphysics that will be fully established in Difference and Repetition - "l'oeuvre souche"- and, in parallel, how this rising issue of the Outside will appeal the complementary issue of Involvement -in others words, the overcoming of Humean physicalism.

Keywords: Hume, Deleuze, Empiricism, Subjectivation, Habit.

\section{Introducción}

El propósito del presente trabajo es mostrar que Empirismo y subjetividad (1953), lejos de ser "una especie de libro-acto [...] donde se dicen algunas cosas que hay que decir sobre Hume"2, prefigura -bien al contrario- las principales secuencias de la metafísica deleuziana: una primera teoría de las síntesis, el esbozo de un primer Afuera, el hincapié en la lógica de las relaciones, el tema de la afectividad como pródromo al pathos radicalizado, etc. Su cometido no consiste, por lo tanto, en valorar la interpretación deleuziana del pensamiento de Hume, sino en recalcar el uso que Deleuze hace del llamado "empirismo radicalizado". En una correspondencia privada ${ }^{3}$, Deleuze explicaba que si se escribe un libro es para: $1^{\circ}$ remediar un error global (función polémica del libro); $2^{\circ}$ reparar un olvido acerca del tema tratado (función inventiva); $3^{\circ}$ crear un concepto nuevo (función creativa). Sentado esto, decimos que el error que combate Empirismo y subjetividad es el de tachar a Hume de haber pulverizado sin más lo dado; el olvido, el dualismo de los términos y de las relaciones; el concepto nuevo, el proceso de subjetivación.

\section{2. ¿Qué es empirismo? El espíritu y su delirio}

Una de las grandes originalidades de Hume reside, según Deleuze, en haber deslindado la cuestión espinosa de la subjetividad, cuestión que, en sus antecesores (Locke, Berkeley), había permanecido encubierta bajo un problema de orden gnoseológico (¿De dónde proceden las ideas? ¿Cuál es su origen?). Desde el inicio de su estudio monográfico, Deleuze procura ubicar la problemática central que guía la

\footnotetext{
2 Cf. Prólogo de O. Masotta a la 3ª edición de Empirismo y subjetividad. Barcelona: Gedisa, 1996.

3 Cf. Villani, A., La guêpe et l'orchidée. Paris: Belin, 1999, p. 56.
} 
investigación filosófica de Hume: “CCómo el espíritu deviene sujeto?” (ES, 13).4 La pregunta, que no es baladí, conlleva ante todo un intento de redefinir el empirismo, rehabilitación semántica sin la cual no podría valorarse correctamente el sentido de la propuesta humeana.

Habitualmente se define esta doctrina "en una relación inversa con el racionalismo" (ID, 213): el empirismo no consistiría más que en una crítica del innatismo, del a priori. Ahora bien, "el empirismo siempre ha tenido otros secretos" (ID, 213), de suerte que no podemos valernos de un principio abstracto (lo Sensible, la Experiencia) para dar cuenta de él. Si queremos alcanzar una definición más satisfactoria que la propuesta por la tradición kantiana ${ }^{5}$, habremos de empezar diciendo que "el empirismo es una filosofía de la imaginación, no una filosofía de los sentidos" (ES, 122). Y vamos a ver que, bien mirado, no es más que otra manera de afirmar que el conocimiento no es lo más importante para el empirismo y que la experiencia no tiene para el empirista -y a mayor abundamiento para Hume- ese carácter unívoco y constituyente que tradicionalmente se le asigna. Lo que interesa al filósofo escocés no es, pues, el origen del entendimiento o de sus contenidos, sino -retrocediendo a un momento anterior- el movimiento por el cual la imaginación deviene facultad, y ello a sabiendas de que el espíritu no posee, en sí mismo, la razón de este devenir.

Una vez descartado el problema abstruso del principio, Hume ofrece el aspecto de esa mala hierba que crece en cualquier hueco, donde no se la esperaba: comienza por el medio, por cualquier sitio. Pero ¿qué significa empezar por el medio en el marco de una ciencia del hombre? A un primer nivel, esto quiere decir que Hume no separa la psicología de otras disciplinas tales como la moral, la economía o la historia. Para el empirista escocés, hay que "ser un moralista, un sociólogo y un historiador antes de ser y para ser un psicólogo" (ES, 12). En efecto, siendo el espíritu lo que es, a saber "colección sin álbum, pieza sin teatro o flujo de percepciones" (ES, 13), carece irremisiblemente de la uniformidad y la constancia requeridas para ser objeto auténtico de una ciencia. Es preciso, por el contrario, que algo - que no dependa de él- lo naturalice, lo califique. El espíritu debe ser afectado para que se dé la posibilidad de un conocimiento en general, razón de por qué Hume invoca la naturaleza humana -el primer Afuera que, al decir de Deleuze, haya sido tematiza-

\footnotetext{
${ }^{4}$ Citamos las obras de Deleuze de acuerdo con las siguientes ediciones y siglas: Empirismo y subjetividad. Barcelona: Gedisa, 1996 = ES; Nietzsche y la filosofia. Barcelona: Anagrama, $1971=$ NF; La filosofia crítica de Kant. Madrid: Cátedra, 1997 = FCK; Proust y los signos. Barcelona: Anagrama, 1995 = PS; El bergsonismo. Madrid: Cátedra, 1987 = B; Diferencia y repetición. Buenos Aires: Amorrortu, 2002 = DR; Lógica del sentido. Barcelona: Barral, 1970 = LS; El Anti Edipo. Barcelona: Paidós, 2004 = AE; Diálogos. Valencia: Pre-Textos, 1980 = D; La isla desierta y otros textos. Valencia: Pre-Textos, 2005 = ID; Deux régimes de fous. Paris: Minuit, 2003 = DRF.

5 Teoría según la cual el conocimiento no sólo empieza con la experiencia, sino que además deriva de ella.
} 
do en la llamada "filosofía del sujeto". Su papel consiste precisamente en calificar la imaginación mediante las afecciones de orden social $y$ pasional. Y si bien es cierto que la pasión no constituye más que una parte de la naturaleza humana -coincidiendo la otra con la asociación de ideas-, habremos de reparar en que el verdadero sentido del entendimiento no es sino, según Hume, volver sociable una pasión y volver social un interés. Entendimiento y pasión parecen formar dos sistemas distintos y separables, pero en última instancia es el segundo dominio quien preside y determina la constitución del primero como materia filosófica: "es porque el hombre tiene pasiones que él asocia sus ideas" (ES, 63).

Si Empirismo y subjetividad muestra que el espíritu afectado constituye el origen común a los dos sistemas del entendimiento y de la pasión, Diferencia y repetición retoma esta idea para llevarla aún más lejos: el pensamiento tan sólo se vuelve efectivo bajo el influjo desgarrador de "algo en el mundo que fuerza a pensar" (DR, 215). El acto rutinario de reconocer, sintomático de un pensamiento que reflexiona, pero que aún no sabe pensar, cede ante una violencia primordial, elemento concomitante -y no constituyente- de un "poder de afectar y de ser afectado"6, de una "alta tonalidad del alma", auténtica Stimmung que se presenta "como emoción material, constitutiva del pensamiento más alto y de la percepción más aguda" (AE, 28). Asimismo, la noción de encuentro puede ser fructuosamente invocada de cara al engendramiento involuntario del pensar. Pues como Proust ha sabido mostrar: "Es el azar del encuentro quien garantiza la necesidad de lo que es pensado" (PS, 26). En este sentido, contingencia del encuentro y violencia adyuvante apuntan a un afuera del pensamiento más radical aún que aquél presupuesto por el modelo dogmático de la recognición. El mundo exterior, impasible y neutro, no es efectivamente más que la imagen invertida y neutralizada de un Afuera absoluto, el límite relativo de un campo afectivo "separado de aquello que puede" (NF, 89). En otras palabras, la representación no estructura de modo apriorístico el campo de la percepción, sino que tan sólo constituye la forma bajo la cual este campo se halla subordinado a un proceso de clausura (diversidad homogénea) y de interiorización (verdad como exterioridad formalmente poseída: inneidad de la idea, a priori de los conceptos). Dicho esto, el carácter inestable de lo real, en el sentido en que es devenir, arruina por anticipado toda pretensión de acceder formal $y$ materialmente a la verdad. Es la estupidez como "estructura del pensamiento": "no es una forma de equivocarse, expresa por derecho el sinsentido del pensamiento" (NF, 148). Acontecimiento y estupidez, he aquí dos razones suficientes para bascular de una lógica del "es" y del saber hacia una lógica del " $y$ " y de la creencia, "nueva base del conocimiento" de la que Hume es el primer artífice.

\footnotetext{
6 Zourabichvili, F., Deleuze. Une philosophie de l'événement. Paris: PUF, 2004, p. 44.

${ }^{7}$ Klossowski, P., Nietzsche y el círculo vicioso. Buenos Aires: Altamira, 1995., p. 11.
} 
Más aún, antes del "yo creyente", existe -y le pervive- un espíritu delirante. De hecho, la objetivación del mismo no agota los poderes de la fantasía. Mientras el espíritu gana en constancia de cara a los términos-percepciones que se asocian en él, la fantasía adquiere tendencia, tendencia a la ilusión. Tal es la "paradoja coherente de Hume": "la imaginación no puede devenir en sí una naturaleza sin seguir siendo para sí una fantasía" (ES, 16). No hay, pues, conocimiento alguno que no se recorte sobre el fondo dionisiaco de un delirio primitivo, "superficie de calma recuperada en la que flotan determinaciones no ligadas, como miembros dispersos, cabeza sin cuello, brazo sin hombro, ojos sin frente" (DR, 61).

Hume nos dice ante todo que el espíritu no tiene los caracteres de un sujeto constituyente. Su crítica es empirista en el sentido fuerte de la palabra: a diferencia del dogmatismo, pero también de la filosofía trascendental, rehúsa partir de la certidumbre esencial de un sujeto previo para preguntarse cómo algo le puede ser dado. Aquí "lo dado ya no está dado a un sujeto; el sujeto se constituye en lo dado" (ES, 93). Ahora bien, el empirista escocés postula de entrada la identidad del espíritu y de sus contenidos, de tal modo que la idea no es aquello por medio de lo cual el espíritu asiste a algo, sino este asistir tomado inmediatamente. ${ }^{8}$ Dicho de otra manera, la imaginación es idéntica al movimiento azaroso de las ideas, al flujo de lo dado en la experiencia.

Pero ¿cuál es el sentido de lo dado? "Es lo que aparece, el ser igual a la apariencia; es el movimiento, el cambio sin identidad ni ley. Se hablará de imaginación, de espíritu, designando por ello, no una facultad, no un principio de organización, sino un conjunto como ése, una colección como ésa" (ES, 93). Por todo ello, la imaginación no puede cumplir la función de determinación determinante; es un determinable. Nada se hace por ella, todo se hace en ella, siendo esa preposición la expresión metafórica de un lugar que está por fijar, y de una "actividad" que no difiere ni un ápice del movimiento de la idea en el espíritu. A este nivel no hay nada más que fantasía, delirio, o, en un vocabulario corregido más tarde por Deleuze, vagabundeo esquizofrénico tendiente a la abolición. El espíritu se presenta a la vez como el objeto de una crítica necesaria y el término de una referencia obligada. Como dice Deleuze: "Hume no hace una psicología atomista; muestra en el atomismo un estado del espíritu que no permite una psicología” (ES, 18). La ciencia del hombre habrá de ser, por consiguiente, una psicología de las afecciones del espíritu.

\footnotetext{
8 Triple identidad del espíritu, de la impresión y del objeto exterior que hace decir a Husserl: "[el espíritu en Hume] produce el mundo entero, el mundo mismo, y no sólo [...] una imagen”. Cf. Husserl, E., La crisis de las ciencias europeas y la fenomenología transcendental. Barcelona: Crítica, 1991, p. 94.
} 


\section{El problema empírico "en estado puro": subjetivación y praxis}

La imaginación, en tanto que "colección de elementos separados", carece aún, en su estadio original, de relaciones fijas que organicen los elementos de percepción y conocimiento del mundo. Hace falta fijar las relaciones por las cuales el espíritu transita de un término a otro; estabilizar lo ligado ${ }^{9}$, para que el espíritu adquiera, de cara a sus contenidos, una generalidad indispensable que no tiene por sí mismo. Sin embargo, lo dado - tal como queda definido en su primera acepción- no contiene la razón de esa objetivación del espíritu. Es preciso buscarla del lado de la naturaleza humana, es decir de lo que también está dado, pero de otra manera, en otro plano. Empezamos a vislumbrar que lo dado no tiene para Hume un carácter unívoco.

Lo dado remite por una parte a la colección de percepciones distintas, pero designa también por la otra a las relaciones -que no dependen de las ideas- y a la subjetividad -que supera a la experiencia. Por tanto, la constancia y la uniformidad de las ideas estriban tan sólo en la manera en que éstas se encuentran ligadas en la imaginación por los principios de asociación (contigüidad, semejanza y causalidad), de suerte que la subjetividad ha de localizarse, en el espíritu, como el efecto de principios que, digámoslo de una vez por todas, son exclusivamente físicos; pues superan al espíritu y lo fijan, pero sin rebasar el marco de la naturaleza humana. Y a este respecto, lo que fundamentalmente interesa a Hume no es la causa de estos principios -origen que, por lo demás, nos es incognoscible-, sino su efecto sobre la imaginación. "La filosofía como ciencia del hombre no tiene que buscar causas; debe escrutar efectos" (ES, 15).

Sentado esto, puede plantearse el problema del Quid facti? El hecho empírico -el único para Hume- es que el sujeto afirma más de lo que sabe y que su juicio supera a la experiencia. Sólo hay un contenido asignable a la idea de subjetividad: la mediación o trascendencia. En este campo de actos noéticos, que "ya no es exactamente el del conocimiento, sino más bien el de la 'creencia' como nueva base del conocimiento" 10 , el sujeto empírico transita, conforme a los principios de asociación, de una parte dada en la naturaleza a otra no dada. Cree, es decir infiere de una idea que le es presente el objeto de otra ausente. "Las palabras 'todos', 'siempre', 'necesariamente' o incluso 'mañana', no remiten a nada de la experiencia: no derivan de la experiencia, aun cuando se apliquen a ella", pero cuando creemos y hacemos uso de esas palabras "decimos más que lo que nos es dado, excedemos los datos de la experiencia" (FCK, 27). Ahora bien, Hume considera -y éste es un elemento crucial para Deleuze- que las representaciones son incapaces de dar cuenta de estas relaciones. Siempre nos podemos formar una idea general, pero nada en esta idea

\footnotetext{
${ }^{9}$ La relación es lo ligado, no lo que liga; es una percepción de la imaginación, no una conclusión del espíritu. Cf. ES, p. 17.

10 Cf. "Réponse à une question sur le sujet" en DRF, p. 326.
} 
nos permite explicar por qué la generalidad es, por ejemplo, una relación. ${ }^{11}$ En otros términos, "la asociación es una cualidad que une a las ideas, no una cualidad de las ideas mismas" (ES, 14).

Llegados a este punto, comprendemos el desarrollo desigual que afecta a la doble inspiración humeana. Si el atomismo constituye la teoría de las ideas en cuanto las relaciones les son exteriores, -razón de por qué una psicología del espíritu es imposible-, corresponderá al asociacionismo, cuya "sutileza [es] irremplazable" (DR, 121), ser la teoría de las relaciones, ya que éstas en absoluto son derivables de la naturaleza de las cosas. He aquí el pensamiento en estado de exterioridad y el sujeto como una suerte de "constructo" a partir de lo dado. Pero entre el flujo ilimitado de percepciones separadas (mens momentanea) y las ideas sistematizadas de una subjetividad constituida -o según la terminología corregida de Diferencia y repetición, entre el en-sí de la repetición material que "se deshace a medida que se hace" y el para-nosotros de la representación que no la deja ver-, se halla la diferencia que el espíritu contemplativo sonsaca a ese "entre-dos": la relación como fruto de una pretensión -el para-sí de una síntesis pasiva- que, en un estado de cosas $\mathrm{AB}$, lleva la imaginación a esperar la aparición de B cuando aparece A. Para Deleuze, la relación nos hace retroceder a un momento anterior a la representación: es el correlato de una síntesis pasiva del espíritu que antecede a -y sobre la que se fundan- las síntesis activas de la conciencia. Pero volviendo a Hume, y teniendo en cuenta lo dicho, vemos que su crítica dista mucho de atañer a las relaciones. Se trata -bien al contrario- de una crítica de las representaciones, "justamente porque éstas no pueden presentar a las relaciones" (ES, 22). Así pues, el error del racionalismo consiste en hacer de la representación el criterio para introducir en la idea lo que no se deja constituir en el primer sentido de la experiencia: el contenido de las palabras como "siempre", "cada vez que", etc. Resumiendo, abogar por una teoría de las relaciones es considerar, en un sentido muy cercano al de Whitehead cuando se refería a lo universal, que el sujeto ya no puede cumplir la función de principio explicativo; es lo que debe ser explicado.

Hume nos muestra que el espíritu, lejos de ser sujeto, está involucrado en un auténtico proceso de subjetivación. Asimismo, la subjetividad ha de definirse "por un movimiento y como movimiento, movimiento de desarrollarse a sí misma" (ES, 91), de suerte que queda superada una falsa alternativa. Ni pasivo ni activo, el sujeto está activado: "se vuelve cada vez más activo y cada vez menos pasivo [...]. Digamos, para hablar como Bergson, que el sujeto es ante todo una impronta, una

11 Cf. DR, p. 21: "los empiristas no se equivocan cuando presentan la idea general como una idea particular en sí misma, con la condición de agregarle la presunción de poder reemplazarla por cualquier otra idea particular que se le asemeje en relación con una palabra" [la bastardilla es nuestra]. La relación, como ha mostrado Butler, es indisociable de una pretensión, una suerte de auto-satisfacción práxico-vital ajena a todo orden lógico. 
impresión dejada por los principios, pero que progresivamente se convierte en una máquina capaz de utilizar esa impresión" (ES, 125). Ahora bien, este movimiento de desarrollo es doble. El sujeto no sólo cree, también inventa. Al sistema del entendimiento, a la asociación hace falta añadir, como ya hemos señalado, aquello sin lo cual no tendría sentido hablar de naturaleza humana: el sistema de la pasión y de la moral. La cuestión es, pues, "considerar al hombre primordialmente como nacido para la acción". 12

El conocimiento en Hume no es efectivamente más que el medio de una actividad práctica, razón de por qué no basta asentar relaciones estables entre ideas. También es preciso dotarlas de un sentido, puesto que la acción no soporta semejante ausencia de inclinación: ella busca la utilidad13, "definiéndose lo útil [...] por su disposición 'a promover un bien"" (ES, 139). En otras palabras, ya no se trata de rodear de relaciones fijas el espíritu, sino de imponerle "centros de fijaciones". Incumbe, pues, a los principios de la pasión vivificar la tendencia por la cual el espíritu afectado considera las impresiones de placer como un bien a proseguir, y las impresiones de aflicción como un mal que hay que evitar. De hecho, el hombre, en tanto manojo de pasiones por las que establece relaciones de simpatía o de aversión, necesita crear medios oblicuos e indirectos de satisfacción para hacer partícipes, dentro de una totalidad moral no preexistente, a partes que ya no son meramente extensivas, sino mutuamente excluyentes. El artificio de la institución constituirá, en este sentido, el único medio positivo para no recaer en lo que es el mal por antonomasia, a saber: la contradicción natural de las parcialidades. Y si bien la tendencia adquirida bajo el efecto de los principios puede extraviar la imaginación en el campo del conocimiento (creencias ilegítimas), ocurre todo lo contrario en el ámbito de la moral: la imaginación, al tiempo que reflexiona los límites impuestos por los principios de la naturaleza humana, se libra de ellos y logra rebasar correlativamente la parcialidad afectiva que le era propia. Así es como, en Hume, lo más frívolo se codea con lo más serio: "la ilusión de la fantasía es la realidad de la cultura" (ES, 61).

En este punto, nos encontramos de nuevo con lo que decíamos al principio, a saber que el sistema del entendimiento se halla subordinado a la actividad práctica y moral. Pues "para que haya un problema de la razón [...] es menester que haya un dominio que escape a la razón y la ponga en cuestión" (ES, 25). La asociación de ideas depende en última instancia de un "asunto de circunstancias": se trata de la afectividad. El sujeto se instituye en y para la práctica, de modo que los factores circundantes son a la subjetivación lo que la pasión es a la asociación: su razón suficiente, lo que confiere al sujeto como estructura posible una existencia, pero "una

12 Hume, D., Investigación sobre el conocimiento humano. Madrid: Alianza, 2007, p. 27.

13 Para Hume lo útil no atañe tan sólo a las cosas, concierne también a las pasiones, a los sentimientos y a los caracteres. 
vez aclarado -dice Deleuze- que lo real precede a lo posible" (ES, 134). Si los principios de asociación cumplen, pues, una función de universalización, han de ser forzosamente los principios de la pasión quienes singularicen al sujeto y le asignen un contenido particular que le es estrictamente esencial. En consecuencia, la aparente dualidad del entendimiento y de la moral ya no recorta exactamente el par disyuntivo teoría/práctica. La única teoría posible ha de ser "teoría de la práctica": el cálculo de las probabilidades para el entendimiento; la justicia para la moral.

El hecho de que la asociación de las ideas dé solamente cuenta de la "corteza" -"sólo explica en nosotros lo superficial, el formalismo de la conciencia" (ES, 113)-, así como el que sea la circunstancia quien le proporcione a la relación su razón suficiente, esto, dice Deleuze, significa que "la subjetividad es, en su esencia, práctica" (ES, 115). Tal es la unidad definitiva de las relaciones mismas y de las circunstancias: "las relaciones medio-fin y motivo-acción son, sí, relaciones, pero también algo más" (ES, 115). En resumidas cuentas, el sujeto se presenta en Hume como lugar provisional de un proceso de habituación en donde se imbrican tres planos heterogéneos: $1^{\circ}$ el plano de los términos inconexos; $2^{\circ}$ el de las relaciones que pueden variar sin que varíen los términos; $3^{\circ}$ el de las circunstancias que hacen variar las relaciones.

Empirismo y subjetividad levanta acta del carácter práctico de la subjetividad y empieza a reparar en la inextricable tensión que, ya en Hume, media entre el Yo [Je] universal y el mí mismo [moi] individual. Diferencia y repetición se propone, por su parte, desembrollar ese "mixto mal analizado" que es el sujeto; pues éste, en tanto que es efecto y no causa, residuo y no origen, constituye una suerte de obstáculo epistemológico cuyas articulaciones reales han de ser descubiertas. Uno de los hallazgos de Diferencia y repetición, motivado en parte por la teoría humeana de la afección, reside en que el Yo $[\mathrm{Je}]$ se inaugura en un "Yo siento más profundo" que las vivencias cotidianas del mí mismo [moi], "emoción verdaderamente primaria que en un principio no siente más que intensidades, devenires, pasos" (AE, 27). El sujeto, "sin identidad fija, siempre descentrado, deducido de los estados por los que pasa" (AE, 28), transita de una disyunción inclusiva que lo instaura, a una identificación exclusiva que lo separa radicalmente de lo que deviene. En otras palabras, ni la forma del Yo $[\mathrm{Je}]$ ni la materia del mí mismo [moi] nos informan acerca de la modalidad de individuación. Todo empieza con "acontecimientos incorpóreos en la superficie" (LS, 15) de una sensibilidad primaria, sopa a la vez pre-subjetiva y preobjetiva acerca de la que la imaginación humeana nos da, como se mostrará en breve, una primera indicación. Sucesivamente deducido de la sumatoria de intensidades por las que pasa y siente que deviene otro, el sujeto es fundamentalmente retrospectivo: al igual que la lechuza de Minerva, llega siempre tarde.

Mientras Hume nos invita a operar una recesión, ya no hacia un ego cogito, sino hacia una "placa sensible" desprovista de la razón de su activación, Deleuze desa- 
rrolla todas las implicaciones del problema empírico: la desubjetivación no tiene por único cometido poner al sujeto teórico entre paréntesis; debe liberar también la multiplicidad de yoes embrionarios que, por persistir intensivamente más acá del Sujeto, son los únicos capaces de aguantar las sacudidas de los devenires, y que, al serlo, posibilitan correlativamente la vida del Yo global e integrador. Pues "bajo el yo que actúa, hay pequeños yoes que contemplan y que vuelven posibles la acción y el sujeto activo" (DR, 126-7).

\section{Experiencia real: heterogeneidad y divisibilidad}

Hemos insistido sobremanera en el problema que plantea Hume: ¿cómo se configura un sujeto en lo dado? A esa cuestión corresponde una crítica de la cuestión que, como tal, la posibilita y le es inherente: las relaciones son exteriores a los términos, siendo el atomismo y el asociacionismo sus implicaciones necesarias. Una vez posicionado el problema empírico, Deleuze repara en que muchas de las objeciones que se les dirigen a los grandes filósofos, y en este caso a Hume, no tienen de filosóficas más que el nombre: Hume habría pulverizado sin más lo dado; su filosofía denotaría un gusto peculiar acorde al espíritu de la época, y además ello sería razón suficiente para criticar su sistema y redargüir que "las cosas no son así". A raíz de esas malas objeciones -critican una teoría sin considerar la naturaleza del problema al que responde y en el que halla su fundamento y estructura-, Deleuze esboza una primera versión de lo que habrá de convertirse, a partir de Nietzsche y la filosofía, y especialmente de El bergsonismo, en un tema recurrente de su filosofía: la teoría de los problemas.

Para Deleuze, es obvio que los factores psico-sociológicos pesan inevitablemente sobre la manera de plantear un problema. Pero, en el fondo, lo que se vuelve problema no es sino aquello que se impone al filósofo como necesidad, como falta radical de elección. Hay algo en el mundo que fuerza a pensar, y el problema, en cuanto creación del pensamiento violentado, es correlato de un encuentro contingente. Si una objeción del tipo "las cosas no son así" adolece de cierta inanidad, es porque el mundo no posee el carácter fiable que habitualmente le prestamos. En rigor, la cuestión no es saber si las cosas son así o no lo son (postulado dogmático de un mundo neutro), sino saber si es riguroso o no el problema que las hace así (intento por igualarse al acontecimiento planteando un buen problema). Paralelamente a su empresa de desmitificación de la identidad personal, Hume es quien primero nos encomienda renunciar a la idea de "realidad exterior objetiva". La sustituye por un mundo de ficción, poblado por criaturas extrañas, pero revelándonos finalmente que este mundo es el nuestro y esas criaturas, nosotros mismos. Tal es el auténtico mundo empirista: "un mundo de exterioridad, un mundo en el cual el pensamiento mismo se encuentra en una relación fundamental con el Afuera, un mundo en el que hay tér- 
minos que son verdaderos átomos y relaciones que son genuinas travesías externas, un mundo en donde la conjunción ' $y$ ' destrona la interioridad del verbo 'ser', mundo de Arlequín, abigarrado y hecho de fragmentos no totalizables en donde la comunicación procede mediante relaciones exteriores" (ID, 214).

Si Hume marca un hito en filosofía al mostrar que subjetividad y verdad son constitutivas de una sola y misma cuestión, induce también otra inflexión significativa: pues, pese a que su fisicalismo se lo impida, invita de algún modo a remontarse hacia una instancia "superior" a la propia verdad. Siguiendo la línea secreta que pasa por Hume, Nietzsche y Bergson, Deleuze muestra, conforme a un "kantismo remodelado [...] o transformado"14, que los caracteres de la Idea recortan precisamente los de esa instancia "superior" que acabamos de mencionar. "Las Ideas, nos dice de acuerdo con Kant, son esencialmente "problemáticas"” (DR, 257). La cuestión consiste, pues, en introducir la diferencia en la verdad misma, siendo las Ideas los elementos internos y genéticos que permiten evaluar las verdades o las concepciones subyacentes de lo verdadero: "Se conocen pensamientos imbéciles, discursos imbéciles construidos totalmente a base de verdades; pero estas verdades son bajas" (NF, 148). Siempre tenemos, pues, las verdades que merecemos en función de los problemas que planteamos, y todo el contexto indica que plantear un problema no es simplemente descubrir, sino inventar. Mientras el pensador ha de hacerse paciente por un lado -encontrarse con el afuera es ser involuntariamente afectado por una emisión de signos-, debe emprender por el otro un esfuerzo por suscitar los términos notables mediante los cuales el problema se planteará. Asimismo, un problema emerge verdaderamente cuando el pensamiento que lo plantea es forzado, cuando padece el efecto de una violencia exterior. De ello deriva, como aparece en Deleuze, una tipología de los valores de verdad: existen verdades bajas (reconocimientos exactos) y verdades altas (posiciones de problemas); e inversamente, hay falsedades bajas (errores en el reconocimiento) y falsedades altas (falsos problemas).

Tras rehabilitar la doctrina de las facultades - pero dirigiéndola secretamente a otro espíritu- y mostrar que las Ideas no se corresponden exclusivamente con la razón, sino "una por una con todas las facultades" (DR, 292), Deleuze rendirá un homenaje implícito a Hume: las Ideas, en tanto que problemáticas y problematizantes, no constituyen sino la trama de una "mecha explosiva" que, desde la sensibilidad (el espíritu como placa sensible) y su objeto propio (una peculiar afección) hasta los contenidos más específicos del pensamiento, activa a todas las facultades. ${ }^{15}$ Cada una de ellas es entonces empujada hasta su más extremo límite y entra

14 Lebrun, G., "Lo trascendental y su imagen" en Gilles Deleuze. Una vida filosófica. Santiago de Cali (Colombia): Revista "Sé Cauto", 2002, p. 91. Véase al respecto el uso notorio que Deleuze hace de Nietzsche y de Bergson.

15 Cf. DR, p. 294: "el pensamiento sólo está determinado a captar su propio cogitandum en la extremidad de la mecha violenta que, de una Idea a otra, pone en movimiento, en primer lugar, la sensibilidad y su sentiendum". 
en un ejercicio trascendente, el cual disuelve de improviso el sentido común, es decir, el ejercicio empírico de facultades que recaen de forma armoniosa sobre un objeto supuestamente uno e idéntico. Asimismo, emerge el "para-sentido": un signo, un elemento heterogéneo irrumpe en el campo de los objetos reconocidos y de las significaciones homogéneas, y pone en movimiento a todo el pensamiento. Tal es el principio de una lógica del sentido, lógica que se encuentra ya esbozada en Empirismo y subjetividad. En efecto, si el cometido de una teoría filosófica no consiste "en resolver un problema, sino en desarrollar hasta el fondo las implicaciones necesarias de una cuestión formulada" (ES, 118), esto significa, en un vocabulario posteriormente corregido, que el par pregunta/respuesta no tiene sentido (y valor) más que en función del problema que lo origina. De ahí que el elemento del pensamiento ya no sea la verdad. Como dice Deleuze: "no hay crítica de las soluciones, sino sólo una crítica de los problemas" (ES, 118). Conforme a esta metodología, vemos que si se le quiere objetar algo a Hume, es necesario juzgar el problema preciso que él plantea, y nada más.

El rasgo fundamental del empirismo, y de la filosofía íntegra de Hume, radica en el dualismo términos/relaciones. Cuando decíamos que Hume se asemeja a esa mala hierba que inesperadamente crece en cualquier hueco, distinguíamos varios niveles. El segundo nivel, más profundo, atañe a las relaciones mismas: también éstas se ubican el medio, en cualquier hueco, y sólo existen como tales relaciones, sin necesitar más base. No son objeto de una representación, sino "medios de una actividad". Asimismo, no pueden originarse en este "caos" impresional que es el espíritu desvaneciente; están dadas en otro plano, en el plano físico de la naturaleza humana -y tal es, según Deleuze, el motivo por el cual Hume logra recusar al tiempo los problemas de génesis psicológica y de deducción trascendental. Pero a pesar de la separación aparentemente insolventable entre términos-percepciones y relaciones, no deja de ser llamativo el parecido que se encuentra entre el espíritu humeano y el "plano de inmanencia" tematizado en Spinoza y el problema de la expresión. Siendo idéntico a sus impresiones, y no habiendo nada que las haya introducido en él, el espíritu se aproxima en cierta medida a la descripción de una inmanencia absoluta: pues no es accidente de una Sustancia ni modificación de un Sujeto. Y no obstante, algo le impide volverse plenamente inmanente a sí mismo.

Hume, en razón de su fisicalismo, no llega a identificar el espíritu con un campo trascendental descategorializado, pero William James, bajo la exhortación a volver más acá de Kant, lo convertirá en el campo de la experiencia pura en donde lo dado cesa de coincidir con una distribución azarosa de mínimos sensibles (átomos psíquicos), sin quedar atrapado por ello en el atolladero del esquema hilemórfico. "Puro" ya no se dirá en el sentido en que lo dicen los empiristas clásicos de una experiencia que se confunde aún con el momento de la tabula rasa; ni tampoco en el sentido idealista de una determinación de unas formas que recaen apodícticamen- 
te sobre la materia como la condición sobre lo condicionado. ${ }^{16}$ El campo de la experiencia en estado puro será el campo de las cosas fragmentadas (para Deleuze: los simulacros, y posteriormente, los objetos parciales) y de las relaciones dinámicas, sin que exista forzosamente una conciencia de estas relaciones. ${ }^{17}$ Libre de toda forma, designará a una realidad intermedia y exterior a todo hilemorfismo. En una peculiar reiteración del gesto nietzscheano, James operará en el sentido de una radical inversión del platonismo, sustituyendo el esquema materia/forma por la noción de material: ni materia ni cosa informe, el material remitirá a una especie de madeja hecha de relaciones y pedazos heterogéneos, auténtico tejido del que estarán compuestas tanto las realidades físicas como psíquicas. Tal es el "nuevo discurso" del que hablará Deleuze en Lógica del sentido: "ya no es el de la forma, pero tampoco el de lo informe. Es más bien lo informal puro" (LS, 142). Ahora bien, encontramos ya en Hume, sin necesidad de forzar demasiado los textos, unos indicios que anticipan de alguna manera aquello que no es primitivamente ni subjetivo ni objetivo, y que se asemeja, para hablar como el filósofo anglo-americano, al stream of consciousness. Parece, pues, posible establecer una primera distinción entre la experiencia del sentido común y la experiencia pura o real.

En primer lugar, Deleuze muestra que lo dado no supone nada: no es afección de una sustancia, ni tampoco modificación de un sujeto. Por otra parte, podrá redargüirse que lo dado se da al menos a los sentidos, lo cual implica tener órganos "y hasta un cerebro", pero Deleuze advierte que nos está prohibido hacer del organismo el principio psico-fisiológico (de una síntesis) de lo dado. Pues "lo que hay que evitar, ahora y siempre, es asignarle desde luego al organismo una organización que ha de llegarle sólo cuando el sujeto mismo llegue al espíritu, es decir, una organización que depende de los mismos principios que el propio sujeto" (ES, 96). Dicho esto, vemos que la experiencia a la que se refiere Hume dista mucho de agotarse en las vivencias ordinarias: se produce en un "extraño teatro" en el que no hay aún nada, y sobre todo, nadie.

La experiencia real que, por oposición a la vivencia fenomenológica, apunta siempre a un "fenómeno-límite", supone un esfuerzo casi metafísico del pensamiento para captar lo que, hablando anacrónicamente, no se deja aprehender en el ejercicio empírico de las facultades: "jamás el ejercicio ordinario de nuestras facultades (percibir, imaginar, concebir, etc.) permitirá su descripción, ya que no se trata de la forma homogénea de una vivencia que poseemos a priori como forma posible, 'horizonte de conocimiento' o de 'familiaridad' (no hay fenómeno sin tal horizonte, dice Husserl) [...]. El fenómeno-límite no puede entonces darse simplemente a

16 Cf. LS, p. 31: paso extraño "que consiste en elevarnos de lo condicionado a la condición para concebir la condición como simple posibilidad de lo condicionado".

17 Cf. Lapoujade, D., "William James, del campo trascendental al nomadismo obrero" en Gilles Deleuze. Una vida filosófica. Santiago de Cali (Colombia): Revista "Sé Cauto", 2002, p. 113. 
la conciencia; nunca ésta podrá recogerlo en una presencia plena y entera". ${ }^{18}$ Hume nos invita, pues, a operar una recesión hacia un mínimo sensible: el átomo de impresión o "momento del espíritu". Sentado esto, es conveniente hacer una aclaración. Según Deleuze, el principio constitutivo de la experiencia humeana no es: "toda idea deriva de una impresión" (uso meramente regulador), sino: "todo lo separable es distinguible, y todo lo distinguible es diferente" (ES, 94). Tal es el principio de diferencia: lo dado es una sucesión animada de percepciones distintas que existen con independencia de las demás. Pero no es esta discreta revolución humeana, consistente en asimilar ser y apariencia, lo que más interesa a Deleuze.

Lo notable radica en que el principio de diferencia cobra todo su sentido a la luz de la divisibilidad. Ésta "produce la consistencia del espíritu hasta la más pequeña idea, desde entonces indivisible". ${ }^{19}$ Ahora bien, el momento indivisible del espíritu es punto sensible. Ni físico ni matemático, designa sin embargo una existencia real: pues entre la extensión y la no-existencia, hay -como dirá Deleuze- un punto singular, cuya actualidad va precisamente a efectuarse según un doble orden, extensivo y cualitativo. En otras palabras, el mínimo sensible de Hume, al localizarse más acá de la vivencia cotidiana, prefigura de algún modo la temática deleuziana de la intensidad, "desigual en sí" que no se deja explicar en la representación como cosa, sin ser desvirtuado por la extensión en la que se reparte y por las cualidades que lo recubren. Siendo a la vez "lo imperceptible y lo que sólo puede ser sentido" (DR, 345), empieza a aproximarse al carácter intermediario del material (James), o inclusive, al signo proustiano: "más profundo que el objeto que lo emite [...] más profundo que el sujeto que lo interpreta" (PS, 47). Por otra parte, la distribución de los minima sensibles en el espíritu ya no presenta del todo ese rasgo harto anárquico que caracterizaba las concepciones atomistas anteriores. Como muestra Deleuze: "es completamente falso decir que en el atomismo de Hume el todo no es más que la suma de sus partes, ya que las partes, tomadas juntas, se definen, por el contrario, por su modo de aparición temporal y a veces espacial" (ES, 99-100). Pues si bien es cierto que la filosofía de Hume está grávida de pre-copernicanismo -espacio y tiempo están en lo dado, y no a la inversa-, resuena sin embargo por todo lo alto una diferencia esencial: mientras el espacio acompaña a percepciones que tan sólo se dejan captar por la vista y el tacto, el tiempo se halla como cuasi-cualidad en todas y cada una de ellas. Así es como Hume, además de proseguir la atomización del ser parmenídeo, renueva la "Idea fisica" en tanto multiplicidad temporal de elementos objetivos del pensamiento. De este modo, lo dado presenta un doble carácter objetivo: "indivisibilidad de un elemento y distribución de los elementos, átomo y estructura" (ES, 99). Pero si bien "es esencial que el átomo se relacione

\footnotetext{
18 Zourabichvili, F., « Kant avec Masoch » en Majeure: Masoch avec Deleuze. Multitudes, 2006/2, 25, p. 94.

19 Villani, A., op. cit., p. 41.
} 
con otro átomo en el seno de una estructura que se actualiza en los compuestos sensibles" (DR, 279), Deleuze insiste a partir de Nietzsche y la filosofía en que la asimilación del punto sensible al átomo impide dar plenamente cuenta de la estructura (como prefiguración de la Idea-problema).

El átomo, en tanto punto duro, es demasiado independiente como para volver del todo inteligible la noción de relación. Como ya mostraba Marx: "los átomos son, para sí mismos solamente, objeto; así que sólo pueden referirse a sí mismos"; y añadía: "-independientes, encerrados en sí mismos, sin intereses mutuos- en nada se relacionan unos con otros". $20 \mathrm{Si}$ el atomismo, tanto físico como psíquico, se propone dar al ser una pluralidad y una distancia esenciales, e incluso sustituirlo por ellas, resulta que la noción de átomo, en la medida en que no puede "contener en sí misma la diferencia necesaria para la afirmación de semejante relación" (NF, 15), corta el intento de raíz. Concibiendo el atomismo a modo de "máscara para el dinamismo naciente", dice Deleuze: "el concepto [de relación] sólo es coherente si se piensa fuerza en lugar de átomo" (NF, 15). El ser de la fuerza es efectivamente plural, no singular; pues la fuerza se halla ya siempre en relación con otras fuerzas, de suerte que envuelve esta exterioridad (pathos, distancia) que el átomo no deja sino en lo separado. Ahora bien, si definimos a su vez la relación de una fuerza con otra bajo el aspecto de una voluntad (voluntad de poder), tenemos el "principio plástico" que preside la génesis diferencial e interna de los compuestos sensibles o fenoménicos. Y tal será precisamente el punto de vista desde el cual Deleuze, vía el dúo HumeNietzsche, arremeterá contra la Crítica: Kant traiciona su proyecto de crítica inmanente casi a la vez que lo proyecta, puesto que las condiciones que invoca son "principios de condicionamiento, no de génesis interna", razón de por qué "permanecen aún exteriores a lo condicionado" (NF, 129). Por el contrario, la voluntad de poder, en la medida en que determina y es determinada simultáneamente por un mundo de fuerzas impersonales y pre-individuales, cesa de remitir a una "condición condicionante" (estilo kantiano) devolviendo así a la inmanencia su absolutez. "Inmanencia: tal es el momento, ya no en el que la experiencia ordinaria se elevaría hasta sus condiciones para hacer de ellas una experiencia de algún modo trascendental, sino en donde esta elevación trascendental resulta ella misma dependiente de la toma de consistencia de una experiencia 'real' -en otros términos, de la alteración de las condiciones bajo las cuales algo es reconocido posible (estilo deleuziano)". ${ }^{21} \mathrm{La}$ voluntad, en tanto que condición condicionada de las fuerzas, puede entonces invocarse idóneamente para dar cuenta del célebre y malentendido clinamen.

Marx ya insistía en que la declinación, pese al carácter independiente del átomo, se presenta en Epicuro y en Lucrecio como "necesidad interna e insensible" del

\footnotetext{
${ }^{20}$ Marx, C., Diferencia entre la filosofía de la naturaleza según Demócrito y según Epicuro. Caracas: Universidad Central de Venezuela, Dirección de Cultura, 1973, pp. 64 y 94.

21 Zourabichvili, F., Deleuze. Une philosophie de l'événement. Paris: PUF, 2004, pp. 10-11.
} 
mismo. Y, bien mirado, no era más que otra manera de decir: la declinación nada tiene que ver con un cambio de dirección en el movimiento del mínimo sensible; y menos todavía, con una indeterminación en la que se reflejaría una suerte de libertad física. Además señalaba que "tal clinamen no tiene [...] tempore certo". Deleuze retoma esta idea para mostrar que el dinamismo de las fuerzas se produce "en un tiempo más pequeño que el mínimo de tiempo continuo pensable", mientras la imagen, que se desprende de esta "síntesis [interna] del movimiento y de su dirección" y garantiza la percepción del objeto, ocupa "el mínimo de tiempo continuo sensible" (LS, 349). La declinación, es decir el acontecimiento como "redistribución de nuestros puntos sensibles" o "mutación de las condiciones de la experiencia", se corresponde con la dimensión intensiva, la mitad real-virtual -y no meramente posible- de una contracción-contemplación que trae a presencia y vuelve perceptible el objeto. Y si Deleuze considera que no hay dificultad alguna en conciliar génesis y estructura, es porque "basta comprender que la génesis no va de un término actual, por más pequeño que sea, a otro término actual en el tiempo; sino de lo virtual a su actualización, es decir, de la estructura a su encarnación, de las condiciones de los problemas a los casos de solución" (DR, 278-9). De ahí derivan dos consecuencias ineludibles en lo que respecta al desbordamiento de la Crítica por la trilogía HumeNietzsche-Bergson: $1^{\circ}$ que el espíritu como placa sensible no es, en sí mismo, indeterminado (non différencié), sino perfectamente diferenciado (différentié), en la medida en que se confunde con una estructura variable e inagotable 22 de puntos sensibles, cuyas relaciones múltiples son ilocalizables y existen como tales más acá de la representación empírica; $2^{\circ}$ que la cosa, ajena a toda forma de interioridad (esencia), se vuelve signo e implica un sentido (plural) que, por ser irreducible a su contenido explícito (mera descripción), ha de ser intuido en la relación de la cosa con la fuerza de la cual es fenómeno.

\section{Habitus o la posibilidad de vivir}

Cuando hablábamos de la subjetividad empírica, decíamos que lo dado es retomado por y en un movimiento que supera a lo dado. La pregunta que debe plantearse ahora es: ¿cómo el tiempo, de estructura, pasa a ser sintesis? Hemos visto que es menester que la asociación le fije al espíritu una generalidad imprescindible para su esfuerzo teórico, pero también -y por adelantado- que la pasión le proporcione una constancia de cara a la actividad práctica. Ahora bien, ambas clases de contenido -salvo el contenido deducido del uso meramente tautológico de la razón- son indisociables de un aprendizaje, una "formación progresiva" (ES, 66), cuyo princi-

22 Cf. LS, p. 36: "no confundir el acontecimiento con su efectuación espacio-temporal en un estado de cosas". 
pio estriba en el hábito. Sin embargo, sería erróneo reducir la costumbre a un simple modo de obrar cultural e históricamente localizable. Se trata más bien, para hablar como el Bergson de Las dos fuentes, del "hábito de contraer hábitos"23 en tanto que define al hombre como especie inventiva.

Así también es como empieza a fraguarse en Hume una "imagen espiralada de la cultura y la naturaleza", imagen en la que ambas se presentan como los dos términos de una integración "en niveles crecientes de complejidades". De ahí que el hábito siga siendo, de algún modo, instinto. Por ello, resulta inexacto decir que son los hábitos del sujeto quienes permiten asociar ideas. A contrario "son los hábitos quienes $[\ldots]$ configuran en lo dado una subjetividad", "quienes facilitan y producen la contracción que somos". ${ }^{24}$ Sentado esto, Deleuze insiste, conforme a la imagen espiralada que venimos manejando, en la "paradoja del hábito": "consiste en formarse por grados y ser a la vez un principio de la naturaleza humana" (ES, 66). El hábito es, en efecto, un principio de la naturaleza humana, pero esto no quita que haya de formarse gradualmente a partir de la contemplación de casos repetidos en la experiencia. Lo cual no es sino otra manera de decir, pero esta vez en un sentido inverso, que la experiencia también es principio, principio al que el hábito mismo presupone. Asimismo, corresponde a la experiencia seleccionar previamente unas impresiones sensibles, y al hábito constituir -pero solamente después-las impresiones de reflexión, insertándose ambos como todo principio "entre el espíritu y el sujeto" y "haciendo que éstas procedan de aquéllas" (ES, 126). De este modo, el sujeto, como "impresión de reflexión, y nada más" (ES, 125), sufre el efecto de una "pasión calma": la tendencia a esperar.

Tocamos aquí lo que Deleuze considera ser el punto nodal del empirismo humeano. Los principios hunden sus efectos en la densidad del espíritu delirante, de suerte que resuena cada vez más en él el eco de un "dinamismo fundamental" (empuje del pasado e ímpetu hacia el porvenir) a raíz del que se vuelve duración. Dicho esto, podemos contestar la pregunta que se había quedado en suspenso: el hábito, en tanto que "síntesis del pasado y del presente con miras al porvenir" (ES, 100), produce en el espíritu una intra-temporalidad que frena el flujo ilimitado de términos-percepciones, "presente viviente" sin el cual el espíritu-estructura permanecería sumido en una mera sucesión de instantes -pues tal sucesión "no hace el tiempo, sino que lo deshace" (DR, 120). A la inversa, es porque contrae los instantes o las excitaciones puntuales por retención selectiva del pasado y protensión del futuro que el hábito da lugar al tiempo en el presente y prepara correlativamente el terreno a un advenir de la subjetividad. "El hábito, dice Deleuze, es la raíz constitutiva del sujeto [...] el tiempo era estructura del espíritu, y ahora el sujeto se presenta como la

\footnotetext{
23 Bergson, H., Euvres complètes. Éditions du centenaire. Paris: PUF, 1970, pp. 996-7.

24 Pardo, J.-L., Deleuze: violentar el pensamiento. Madrid: Cincel, 1990, p. 23.
} 
síntesis del tiempo" (ES, 100-1, 103). Tal es el momento clave en que el espíritu deviene duración: escapa al reino del puro azar ("caos" de las impresiones), y aflora, en este tiempo pasivamente constituido, a modo de subjetividad naciente, cuya capacidad de ritmar las imágenes (del mundo) se irá afianzando.

Si Empirismo y subjetividad empieza a definir el hábito por este presente en el que se desarrollan nuestras actividades -este presente lo somos más que estamos en él-, Diferencia y repetición repara por su parte en cuatro de sus principales aspectos. En primer lugar, el presente, en la medida en que depende de una capacidad de contracción limitada, ha de ser forzosamente finito: se encuentra irremisiblemente alojado en una abertura que se intercala entre el aflojamiento y la reactivación del poder contrayente (p. ej.: entre la fatiga y la necesidad a nivel orgánico). Pero en segundo lugar, vemos que, pese a ello, el presente se repite periódicamente y que, por lo tanto, no puede haber un solo presente, sino una multiplicidad de presentes variables y vivientes. En cualquier organismo, incluso antes de que lo perciba y lo sienta, coexisten varias duraciones o velocidades relativas; razón que lleva Deleuze a enunciar la regla según la cual "no podemos ir más ligeros que [...] nuestros presentes" (DR, 130). Asimismo, nuestra existencia depende de un armazón temporal compuesto de "miles de síntesis pasivas". La consecuencia de todo esto es, en tercer lugar, que el sujeto es, desde lo orgánico hasta lo reflexivo, una suma de contracciones y, en última instancia, una integración global de yoes larvarios: cada contracción, cada presente variable constituye un medio que, sea como fuere el nivel considerado (orgánico, existencial, etc.), sirve de encuadre para la efectuación de nuestros actos. La noción de medio implica una redefinición del sujeto como lugar provisional y moviente en donde se emiten miles de pretensiones. Como dice Deleuze: "sólo se es lo que se tiene, y es gracias a un tener que el ser se forma aquí [local y parcialmente]" (DR, 132). La identidad y consistencia del Yo [Je] se infieren, por lo tanto, de una sumatoria de hábitos-esperas, razón de por qué "es preciso atribuir un alma al corazón, a los músculos, a los nervios, a las células, pero un alma contemplativa cuyo rol se limita a contraer el hábito" (DR, 124). Pues donde se entabla una pretensión se forma un yo larvado que se llena, como dice Deleuze arriesgando una fórmula de Plotino, de una imagen que no es la suya y de la cual sólo goza, volviéndose, para contemplarlo, hacia aquello de lo cual procede. Tal es la "beatitud de la síntesis pasiva": el yo larvado experimenta una emoción de placer que procede de la contemplación-contracción de unas excitaciones puntuales, las cuales se reparten al azar sobre la superficie de una placa sensitiva primaria.

El Ello freudiano se asemeja notablemente aquí a la idea de espíritu delirante que venimos manejando: “un lugar móvil, un 'aquí y allá' de las excitaciones y de sus resoluciones" (DR, 154) que, para respetar la terminología que venimos empleando, no ha sido aún fijado. Pero el placer, en tanto que resolución instantánea de una diferencia de intensidad local (excitación), no puede convertirse por sí solo en 
el principio rector de la vida anímica. 25 Para que la resolución se torne sistemáticamente posible, es preciso que las diferencias de intensidad, primariamente libres y no ligadas, sean previamente "investidas". Tiene que haber un "más allá" del principio de placer que garantice la catexis sistemática de las excitaciones. He aquí de nuevo Habitus: la ligazón, por la cual una excitación local cualquiera adolece de una resolución sistemática, constituye una verdadera contemplación-contracción, de suerte que "en el nivel de cada vínculo se forma un yo [moi] en el Ello; pero un yo pasivo, parcial, larvario, contemplante y contractante” (DR, 155). Asimismo, cada pequeño yo pasivo liga la excitación, siendo él mismo una excitación ligada. El Ello se puebla entonces de sujetos larvados que constituyen en él el tiempo del presente viviente, a la vez que la apremiante prueba de realidad orienta el proceso secundario de organización de la personalidad (integración de los yoes embrionarios - subjetivación) determinando un objeto real como término de la acción. Pero no por ello, la activación y la objetivación dejan de estirarse respectivamente hacia un foco inactual (yo local y objeto parcial) que, al igual que la fantasía humeana y sus contenidos delirantes, continúa insistiendo de forma subterránea.

Habitus abraza todas las esferas de la vida y del pensamiento, desde lo consciente hasta lo más imperceptible. A la vez pasivo y constituyente, es profundamente ajeno a toda forma de memoria o de entendimiento. Antecede y posibilita las operaciones de la conciencia mediante las cuales tanto el pasado inmediato de la retención como el futuro inmediato de la anticipación se transforman respectivamente en el pasado reflexivo de la observación-recuerdo y en el futuro reflexivo de la previsión -en otras palabras, en las dos dimensiones del presente. Tal es el efecto del hábito: orientar la flecha del tiempo, "plantear el pasado como regla del porvenir" (ES, 102). De hecho, sabemos que la subjetividad empírica es para la práctica precisamente porque el hábito organiza el tiempo como "un presente perpetuo al que debemos y podemos adaptarnos" (ES, 104). Pero por otra parte, se ha realzado también el cariz selectivo que impregna todo proceso de habituación: los términos-percepciones, para retomar las palabras de Leibniz en un sentido inverso, tienden con todas sus fuerzas al presente y a la presencia, pero sólo son retenidos, así como actualizados en los compuestos sensibles, aquellos que mejor se adecuan, según el rasero de lo útil, a las circunstancias y necesidades del momento. ¿Qué ocurre entonces con aquellos términos que no han superado la prueba selectiva? De ahí la cuarta y última reseña: debe de existir -por lo menos- otro modo temporal que rescate aquello que el presente, en su transcurso agujereado, no ha retenido.

Deleuze nos dice que "la mayoría de los caracteres de una duración, de una memoria bergsoniana" (ES, 100) se encuentran ya esbozados en el hábito-espera.

25 Freud, S., Más allá del principio del placer. Madrid: Alianza, 1985, p. 96: “el impulso a elaborar algo impresionante [...] puede llegar a manifestarse primariamente y con independencia del principio del placer". 
Pero esto no quita que el pasado siga siendo en Hume una mera dimensión del presente. En este sentido, el presente perpetuo al que da lugar Habitus permanece todavía muy cercano a la "existencia psicológica"26, y el recuerdo, como acto de traer a presencia lo ya vivido, sólo nos re-presenta un antiguo presente. Percibimos que el pasaje de un medio-presente a otro ha de presuponer forzosamente otro modo temporal, una suerte de "nunca vivido" que insista por debajo de las vivencias y de los estados de cosas correspondientes, y sostenga el aparente "salto" kierkegaardiano que se da de uno a otro. A este respecto, la frase clave de Empirismo y subjetividad es: "al determinar el proceso de las impresiones de reflexión, los principios no desarrollan virtualidades contenidas en las impresiones sensibles; éstas no contienen virtualidad alguna" (ES, 126).

Para Deleuze, el hábito puede prescindir fácilmente de la memoria, y a mayor abundamiento, de esta memoria a la que Bergson asignará un carácter ontológico, puesto que está grávido de factores circundantes que lo empujan a "ir a lo esencial": "[la costumbre] no sólo nos familiariza con todo aquello de lo que hemos disfrutado largo tiempo, sino que además engendra una disposición en su favor y nos la hace preferir a otros objetos, que acaso son más estimables, pero a los que conocemos menos". ${ }^{27}$ Ahora bien, este presente selectivo, que nos encamina hacia las "formas estadísticamente aceptadas" de pensar, sentir y creer, y en el que se despliegan todas nuestras actividades molares, no da cuenta de su propio pasaje. He aquí la primera paradoja de Bergson, cuidadosamente recogida por Deleuze en Diferencia y repetición: el presente "constituye el tiempo, pero pasa en ese tiempo constituido" (DR, 132). La sucesión de los presentes depende de una condición: debe de haber una potencialización entre el antes y el después, un "aumento constante de las dimensiones" (DR, 134). Tal es la asimetría del presente que dura: "ya sea que el presente encierra distintamente la imagen siempre creciente del pasado, ya sea, más bien, que testifica, mediante su continuo cambio de calidad, la carga que uno lleva a sus espaldas, tanto más pesada cuanto más viejo uno se va haciendo".28 Asimismo, Deleuze sentirá la necesidad de invocar una segunda síntesis pasiva, un segundo "más allá": Mnemosine o la gigantesca memoria bergsoniana que redobla el campo del presente y contiene todas las dimensiones (virtualidades) capaces de actualizarse; todo un trasfondo dinámico que insiste imperceptiblemente por debajo de nuestra existencia, de los diversos y sucesivos medios que ésta contrae y en los cuales se desarrolla.

Pero al replantear con Proust la pregunta diferida por Bergson: “¿es posible una exploración (erótica) de lo jamás vivido?”, surgirá en Deleuze la intuición de una tercera síntesis del tiempo donde el futuro tendrá el primado. El tercer modo tem-

${ }^{26}$ Cf. B, p. 56: "Hablando con rigor, lo psicológico es el presente".

27 Hume, D., Tratado de la naturaleza humana. Madrid: Tecnos, 1998, p. 675.

28 Citado en B, p. 51. 
poral no sólo afirmará el presente y el hecho de que suceda a otro (pasado), sino que apelará de algún modo dicha sustitución viendo en ella el destino de todo presente. Tal será la "secreta coherencia" de lo intempestivo: deshacer el agente (presente) y la condición (pasado) en provecho de un producto que, como dice Klossowski, "me excluye en el instante mismo en que lo pienso". ${ }^{29}$ La afirmación del devenir constituirá el meollo del problema ético alojado en una filosofía de la inmanencia que enuncia el perpetuo "desfondamiento" del presente y, con él, de la subjetividad.

\section{Conclusión}

Nos proponíamos mostrar que las secuencias de Empirismo y subjetividad son exactamente las de la metafísica deleuziana, siendo Diferencia y repetición el máximo exponente del llamado "empirismo trascendental". Creemos haber encontrado en la articulación del espíritu delirante, de la exterioridad de las relaciones y del proceso de habituación los elementos germinales a partir de los que Deleuze elaborará una teoría del afuera. La otra vertiente, que, con esta temática, forma el "motor abstracto del pensamiento deleuziano", es la implicación (punto-signo-fuerza, Ideaproblema, campo intensivo, tiempo complicado). En la confluencia de ambos temas, se forjan la mayor parte de los conceptos cuya topología hemos procurado restituir a grandes rasgos. Se trataba también de realzar que la cuestión central de la inmanencia, sesgada por el fisicalismo de Hume, no puede ahorrarse un plano virtual donde lo exterior deja de permanecer en lo separado y se halla envuelto en un tiempo heterogéneo irreductible a la mera presencia.

\section{Referencias bibliográficas}

Alliez, E., Deleuze. Philosophie virtuelle. Paris: Synthélabo, 1996.

Bergson, H., Euvres complètes. Éditions du centenaire. Paris: PUF, 1970.

Butler, S., La vie et l'habitude. Paris: NRF, 1922.

Freud, S., Más allá del principio del placer. Madrid: Alianza, 1985.

Hume, D., Tratado de la naturaleza humana. Madrid: Tecnos, 1998.

Hume, D., Investigación sobre el conocimiento humano. Madrid: Alianza, 2007.

HuSSERL, E., La crisis de las ciencias europeas y la fenomenología transcendental.

Barcelona: Crítica, 1991.

James, W., Pragmatismo. Madrid: Alianza, 2007.

Klossowski, P., Nietzsche y el círculo vicioso. Buenos Aires: Altamira, 1995.

29 Klossowski, P., op. cit., p. 71. 
LAPOUJADE, D., "William James, del campo trascendental al nomadismo obrero" en Gilles Deleuze. Una vida filosófica. Santiago de Cali (Colombia): Revista "Sé Cauto", 2002, pp. 113-114.

Lebrun, G., "Lo trascendental y su imagen" en Gilles Deleuze. Una vida filosófica. Santiago de Cali (Colombia): Revista "Sé Cauto", 2002, p. 91.

MarX, C., Diferencia entre la filosofía de la naturaleza según Demócrito y según Epicuro. Caracas: Universidad Central de Venezuela, Dirección de Cultura, 1973.

Pardo, J.-L., Deleuze: violentar el pensamiento. Madrid: Cincel, 1990.

VILlANi, A., La guêpe et l'orchidée. Paris: Belin, 1999.

ZourabichVILI, F., Deleuze. Une philosophie de l'événement. Paris: PUF, 2004.

Zourabichvili, F., «Kant avec Masoch » en Majeure: Masoch avec Deleuze. Multitudes, 2006/2, 25, pp. 91-94.

Julien Canavera

Departament de Filosofia

Universitat de València

julien.canavera@uv.es 